\title{
Risk factors for Clostridium difficile infection in pediatric inpatients: A meta-analysis and systematic review
}

\author{
Scott Anjewierden $\mathrm{BS}^{1, \mathrm{a}}(\mathbb{0})$, Zheyi Han $\mathrm{BS}^{1, \mathrm{a}}$, Charles B. Foster $\mathrm{MD}^{2}$, Chaitanya Pant $\mathrm{MD}^{3}$ (1) and \\ Abhishek Deshpande MD, $\mathrm{PhD}^{4,5}$ (D) \\ ${ }^{1}$ Cleveland Clinic Lerner College of Medicine, Case Western Reserve University, Cleveland, Ohio, USA, ${ }^{2}$ Center for Pediatric Infectious Diseases, Cleveland Clinic \\ Children's, Cleveland, Ohio, USA, ${ }^{3}$ Division of Gastroenterology, Hepatology and Motility, Department of Internal Medicine, University of Kansas Medical Center, \\ Kansas City, Kansas, USA, ${ }^{4}$ Medicine Institute Center for Value Based Care Research, Cleveland Clinic, Cleveland, Ohio, USA and ${ }^{5}$ Department of Infectious \\ Diseases, Cleveland Clinic, Cleveland, Ohio, USA
}

\begin{abstract}
Objective: To summarize risk factors for Clostridioides (formerly Clostridium) difficile infection (CDI) in hospitalized pediatric patients as determined by previous observational studies.

Design: Meta-analysis and systematic review.

Patients: Studies evaluating risk factors for CDI in pediatric inpatients were eligible for inclusion.

Method: We systematically searched MEDLINE, Web of Science, Scopus, and EMBASE for subject headings and text words related to CDI and pediatrics from 1975 to 2017 . Two of the investigators independently screened studies, extracted and compiled data, assessed study quality, and performed the meta-analysis.

Results: Of the 2,033 articles screened, 14 studies reporting 10,531,669 children met the inclusion criteria. Prior antibiotic exposure (odds ratio [OR], 2.14; 95\% confidence interval [CI], 1.31-3.52) and proton pump inhibitor (PPI) use (OR, 1.33; 95\% CI, 1.07-1.64) were associated with an increased risk of CDI in children. Subgroup analyses using studies reporting only adjusted results suggested that prior antibiotic exposure is not a significant risk factor for CDI. H2 receptor antagonist (H2RA) use (OR, 1.36; 95\% CI, 0.31-5.98) and that female gender (OR, 0.87; 95\% CI, 0.74-1.03) did not play a significant role as a risk factor for developing CDI.

Conclusion: Prior antibiotic exposure appears to be an important risk factor for CDI based on the combined analysis but not significant using adjusted studies. PPI use was associated with an increased risk of CDI. Judicious and appropriate use of antibiotics and PPIs may help reduce the risk of CDI in this vulnerable population.
\end{abstract}

(Received 20 November 2018; accepted 19 January 2019)

Clostridioides (formerly Clostridium) difficile is one of the most frequent causes of hospital-acquired infections in both adult and pediatric patients. ${ }^{1,2}$ The incidence and healthcare burden of $C$. difficile infection (CDI) in the hospitalized pediatric population has increased in the past 20 years, ${ }^{3-6}$ mostly attributed to the emergence of the new, hypervirulent strain B1/NAP1/027. Although most children recover without long-term sequelae, CDI in hospitalized children is associated with increased mortality, length of stay, and hospital $\operatorname{cost}^{5}{ }^{5}$ and it is an independent predictor of subsequent colectomy and discharge to short- or long-term care facility. ${ }^{8}$

In adult patients, CDI is associated with discrete risk factors including advanced age, antibiotic exposure, prolonged

${ }^{\mathrm{a}}$ Authors of equal contribution.

PREVIOUS PRESENTATION: An abstract containing data used in this study was presented at the 2018 CSCTR Midwest Clinical and Translational Research Meeting on April 26, 2018, in Chicago, Illinois.

Author for correspondence: Abhishek Deshpande, Email: abhishekdp@gmail.com

Cite this article: Anjewierden S, et al. (2019). Risk factors for Clostridium difficile infection in pediatric inpatients: A meta-analysis and systematic review. Infection Control \& Hospital Epidemiology, 40: 420-426, https://doi.org/10.1017/ice.2019.23 hospitalization, proton-pump inhibitor use, immunocompromised state, and other medical comorbidities. ${ }^{9}$ In comparison, risk factors for CDI in children are less well-defined. Notably, antibiotic and proton pump inhibitor (PPI) use have only been variably associated with CDI risk in children. ${ }^{10-12}$ Additionally, current understanding of pediatric CDI is further complicated by the fact that up to $70 \%$ of infants $<1$ month and up to 2 years of age are colonized with $C$. difficile but do not develop clinical illness until 12-24 months of age. ${ }^{13}$ Therefore, closer examination of currently available evidence is needed to better understand the significance and implications of potential risk factors for pediatric CDI. The aim of this meta-analysis and systematic review was to evaluate the association of previously identified risk factors with CDI in hospitalized children.

\section{Methods}

All procedures used in this study were consistent with Preferred Reporting Items for Systematic Reviews and Meta-Analyses (PRISMA) guidelines. ${ }^{14}$ 


\section{Data sources and searches}

Two investigators (S.A. and Z.H.) systematically searched the literature independently using the following predetermined inclusion criteria: (1) observational studies (including case control and cohort) evaluating risk factors for primary CDI, (2) pediatric patients ( $\leq 19$ years), (3) study population includes $>10$ pediatric patients, (4) in-patients admitted to a hospital, and (5) studies evaluated $>1$ CDI risk factor. Studies were excluded if (1) they exclusively studied CDI in children $<2$ years, in which the role of $C$. difficile was unclear and testing was not routinely recommended, ${ }^{9}$ and (2) if they exclusively studied CDI patients in an outpatient setting. The following databases were searched from January 1975 to August 2017: MEDLINE (PubMed), EMBASE, Web of Science, and Scopus. The following search terms were used: C. diff infection, Clostridium difficile infection, CDI, Clostridium difficile associated infection, $\mathrm{CDAD}$, pediatric, paediatric, children, infants, adolescents, risk, risk factors, predictor, and marker. We also conducted a modified search from September 2016 to August 2017 including Clostridioides difficile in our search criteria, but we did not find any additional eligible studies (data not shown). The electronic PubMed search strategy is available in the supplemental appendix online.

\section{Study selection and data extraction}

A list of retrieved articles that met the inclusion criteria was reviewed by 2 investigators independently (S.A. and Z.H.). These investigators also independently extracted data from the full text of the included studies. The data collected included study design, study population, patient demographics, clinical characteristics, and identified risk factors for CDI. Any disagreement was resolved in consensus with a third investigator (A.D.). Authors were contacted if relevant information was not available for a particular study. The Cohen's interrater $\kappa$ statistics for inclusion agreement and data extraction were 0.85 and 0.90 respectively, which indicated excellent interrater agreement.

\section{Quality assessment}

The quality of the observational studies (including cohort and case-control) was assessed independently by 2 investigators (S.A. and Z.H.) using the Newcastle-Ottawa Scale (NOS) ${ }^{15}$ Studies with NOS scores $>7$ were considered high-quality studies, and those with NOS scores of 5-7 were considered moderate-quality studies. Any disagreements or discrepancies were resolved by consensus with a third investigator (A.D.). The Cohen's interrater $\kappa$ statistic for study quality assessment was 0.90 , which indicated excellent interrater agreement.

\section{Data synthesis and analysis}

Due to the diversity of risk factors evaluated in studies of C. difficile, we decided a priori that all risk factors reported in $\geq 3$ studies were eligible for inclusion in the meta-analysis.

For all studies, when possible, we extracted the adjusted odds ratios (ORs) and relative risks. When adjusted data were not available, crude odds ratios and relative risks with their $95 \%$ confidence intervals (CIs) were calculated from the number of events.

We decided a priori that adjusted data would be used for all meta-analyses with $\geq 3$ studies. When adjusted data were not available for $\geq 3$ studies, we combined the adjusted and unadjusted data. DerSimonian and Laird random-effects models were used for all meta-analyses. ${ }^{16}$ The meta-analysis was performed using the inverse variance method to obtain pooled ORs and $95 \%$ confidence intervals (CIs). We assumed similarity between the OR and other relative measures, such as relative risk or rate ratios, because of low disease frequency and prevalence of CDI in this population. We evaluated statistical heterogeneity using the Cochran $\chi^{2}$ (Cochran Q) and the $\mathrm{I}^{2}$ statistic. We defined significant heterogeneity as a $\chi^{2}<0.10$ or an $I^{2}$ statistic $>66 \%$. ${ }^{17}$ Moderate heterogeneity was defined as an $\mathrm{I}^{2}$ statistic between $33 \%$ and $66 \%$. Low heterogeneity was defined as an $\mathrm{I}^{2}$ statistic $<33 \%$.

\section{Assessment of publication bias}

To check for publication bias, we generated funnel plots and used Egger's regression asymmetry test. Where asymmetry was detected, we assessed the potential impact of the publication bias using the Duval and Tweedie nonparametric "trim-and-fill" method. ${ }^{18}$

We used Review Manager software (RevMan, version 5.3 for Windows, Oxford, UK; The Cochrane Collaboration, 2014) for our statistical analyses.

\section{Results}

\section{Study characteristics}

The preliminary literature search identified 2,032 publications (Fig. 1). After removing duplicates and screening titles for potentially relevant articles, 127 studies were considered relevant. On further screening of the abstracts of these potentially relevant studies, 56 were selected for full text review. Finally, a total of 14 articles met the full inclusion criteria and were included in the systematic review. The reasons for excluding the remaining 42 articles are listed in Figure 1.

The main characteristics of the included studies are summarized in Supplemental Table 2. The final study population consisted of 10,531,669 children, of which 22,320 patients developed CDI. In analyzing the 14 included studies, 7 were retrospective cohort studies and 6 were retrospective case-control studies, while the remaining study was a prospective cohort study. Six of the studies were conducted in the United States, 2 were conducted in Italy, with a single study was conducted in each of the following countries: Canada, China, Croatia, Japan, Spain, and Turkey. The testing methodology for CDI consisted of a $C$. difficile toxin assay for 10 studies. Clostridium difficile culture and/or toxin assay for 2 studies and the use of the International Classification of Diseases Ninth Edition (ICD-9) code 008.45 or other billing codes were used in the remaining 3 studies.

\section{Quality assessment}

Using the NOS scale, all included studies were identified as moderate or high in quality (Supplemental Table 1 online). Most included studies clearly identified the study population and defined the outcome and outcome assessment. Most studies identified important confounders that were used for adjustment of the association exposures and risk of CDI. We found considerable variation in the selection of available confounding variables for adjustment. A few confounding variables may not have been fully identified and recorded. The most common confounders adjusted were age, gender, and antibiotic exposure. Information on the dose and duration of antibiotic therapy prior to the diagnosis of CDI was limited. Various methods were used to identify antibiotic use, including review of patient medical records, patient prescription records, and ICD-9 codes. 


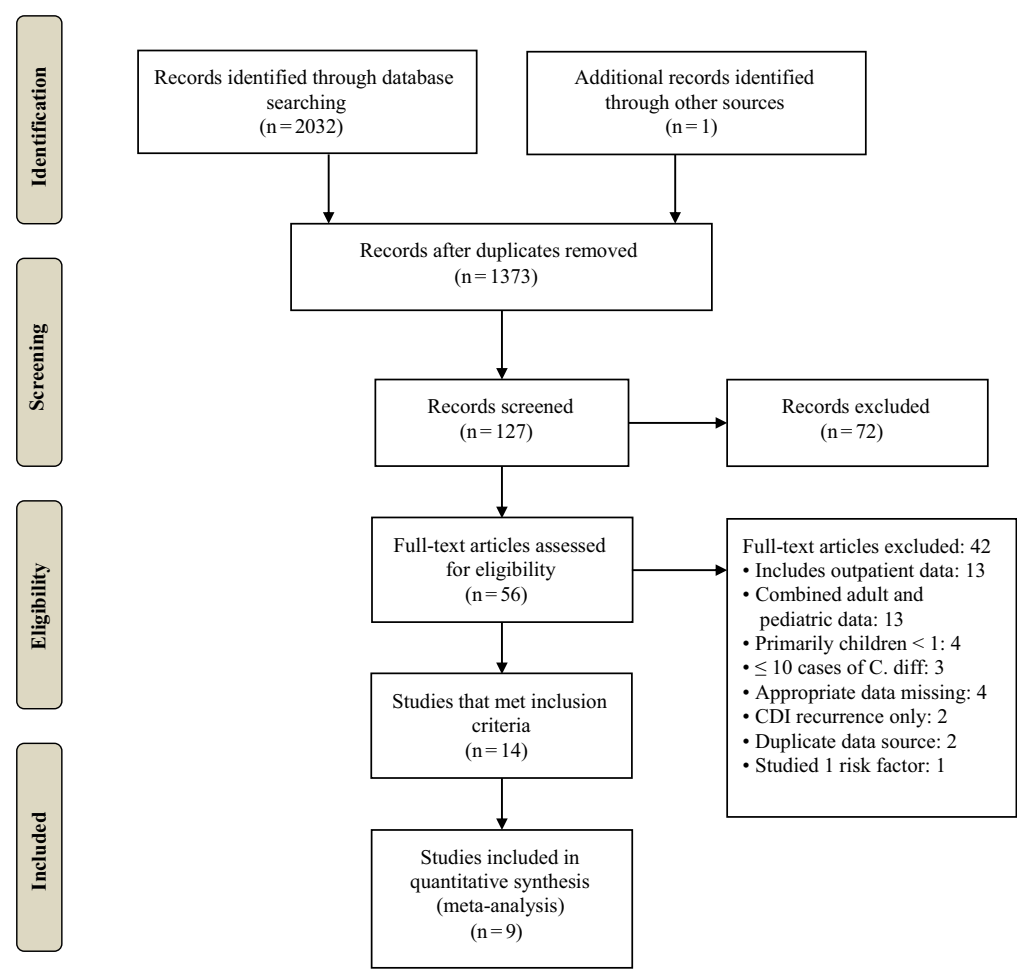

Figure 1. Flow chart for study inclusion in the systematic review and meta-analysis.

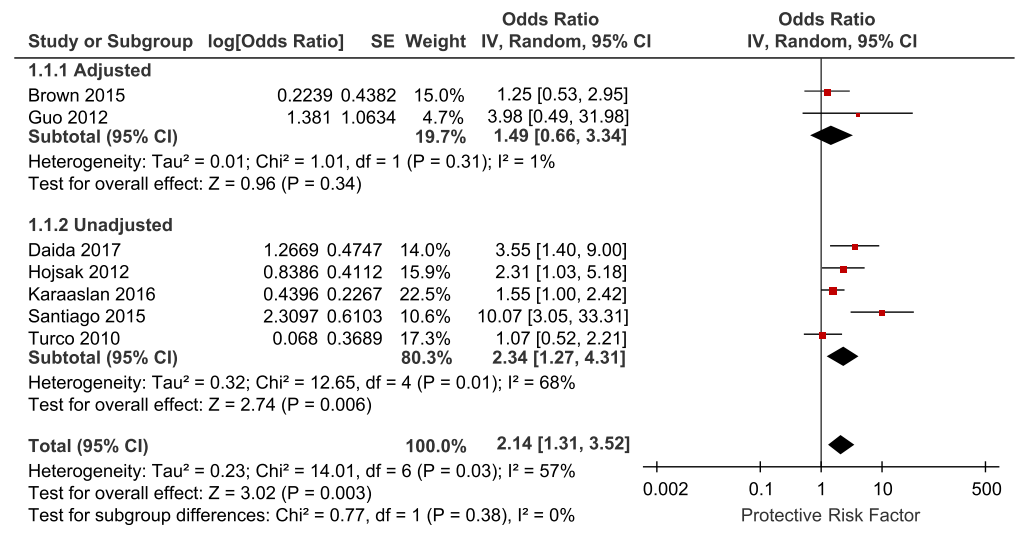

Figure 2. Forest plot of the association between antibiotic use and CDI. Vertical line corresponds to no difference point between the 2 groups. Squares correspond to risk ratios. Horizontal lines represent the $95 \%$ confidence intervals. The diamond indicates the pooled relative risk ratios. Note. df, degrees of freedom; M- $\mathrm{H}$, Mantel-Haenszel.

\section{Meta-analyses of risk factors for CDI}

\section{Exposure to antibiotics}

A total of 7 studies reported data on prior antibiotic exposure ( 5 unadjusted and 2 adjusted studies). Because $<3$ studies provided adjusted data, we combined studies reporting the adjusted and unadjusted data. Meta-analysis of the 7 studies demonstrated a significantly increased risk of CDI with prior exposure to any antibiotic class (OR, 2.14; 95\% CI, 1.31-3.52; $P=.003$ ) (Fig. 2). There was moderate heterogeneity among these studies $\left(\mathrm{I}^{2}=57 \%\right)$. We also performed subgroup analysis for the adjusted and unadjusted studies. The meta-analysis of the unadjusted studies showed a significantly increased risk of CDI with prior exposure to any antibiotic class (OR, 2.34; 95\% CI, 1.27-4.31; $P=.006)$. There was significant heterogeneity among these studies $\left(\mathrm{I}^{2}=68 \%\right)$. The meta-analysis of the adjusted studies also showed an increased risk of CDI with prior exposure to antibiotics, but the results were not statistically significant (OR, 1.49; 95\% CI, 0.66-3.34; $P=.34$ ). There was low heterogeneity between the 2 adjusted studies $\left(\mathrm{I}^{2}=1 \%\right)$. Risk factors for CDI with individual antibiotic subclasses were not reported by $>2$ studies and were therefore not included in the meta-analysis.

\section{Gastric acid suppression}

We identified 4 studies that reported adjusted data on PPI use as a risk factor for CDI. Meta-analysis of the 4 studies showed increased risk of CDI with PPI use (OR, 1.33; 95\% CI, 1.07-1.64; $P=.01$ ) (Fig. 3). There was moderate heterogeneity among these studies $\left(\mathrm{I}^{2}=36 \%\right)$. Adjusted data for the risk of CDI with $\mathrm{H} 2$ receptor antagonist (H2RA) use was obtained from 3 studies. Meta-analysis of the 3 studies examining H2RA use also showed increased risk of CDI associated with H2RA use, but the result was not statistically 


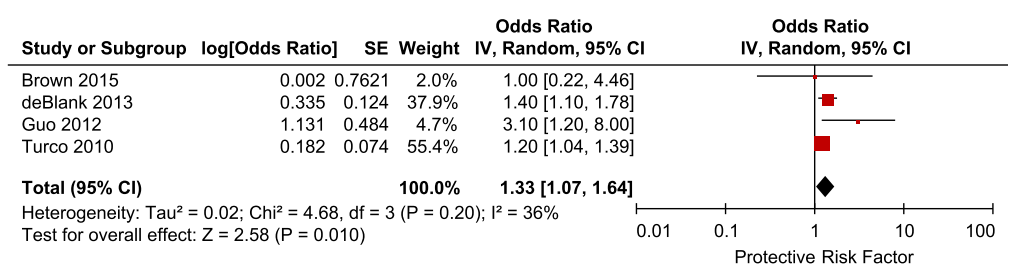

Figure 3. Forest plot of the association between PPI use and CDI. Vertical line corresponds to no difference point between the 2 groups. Squares correspond to risk ratios. Horizontal lines represent the $95 \%$ confidence intervals. The diamond indicates the pooled relative risk ratios. Note. df, degrees of freedom; M- $\mathrm{H}$, Mantel-Haenszel.

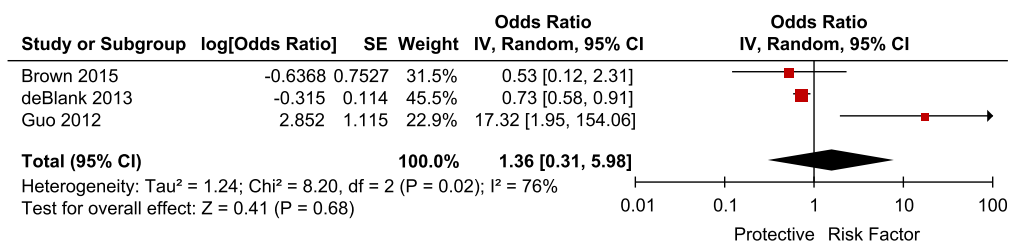

Figure 4. Forest plot of the association between H2RA use and CDI. Vertical line corresponds to no difference point between the 2 groups. Squares correspond to risk ratios. Horizontal lines represent the $95 \%$ confidence intervals. The diamond indicates the pooled relative risk ratios. Note. df, degrees of freedom; M- $\mathrm{H}$, Mantel-Haenszel.

significant (OR, 1.36; 95\% CI, 0.31-5.98; $P=.68$ ) (Fig. 4). There was significant heterogeneity among these studies $\left(\mathrm{I}^{2}=68 \%\right)$.

\section{Gender}

We identified 4 studies that reported adjusted data on gender as a risk factor for CDI. Meta-analysis of the 4 adjusted studies did not show a significantly increased risk of CDI associated with female gender (OR, 0.87; 95\% CI, 0.74-1.03; $P=.10$ ) (Fig. 5). There was significant heterogeneity among these studies $\left(\mathrm{I}^{2}=76 \%\right)$.

\section{Other risk factors}

Several additional risk factors associated with pediatric CDI were not included in the meta-analysis because they were reported in $<3$ studies. These risk factors and their corresponding estimated effect sizes have been listed in Supplemental Table 2 (online). Notably, underlying comorbidities that have been previously reported such as inflammatory bowel disease (IBD), solid organ transplant, and malignancies ${ }^{10,19},{ }^{20}$ were also reported by multiple studies included in this review (Supplemental Table 2 online).

\section{Publication bias}

We did not assess publication bias because there were $<10$ included studies (for each risk factor that was meta-analyzed). A minimum number of 10 studies is suggested when assessing publication bias using a funnel plot or other more advanced regression-based methods. However, we constructed a funnel plot for 2 of the variables, antibiotic exposure and PPI use, because these risk factors had a large number of patients included from 7 and 4 studies, respectively (Supplemental Fig. 1 online).

\section{Discussion}

In this meta-analysis of 14 studies, prior antibiotic exposure and PPI use were significantly associated with increased risk of developing CDI. Children with prior antibiotic exposure have approximately twice the risk of developing CDI compared to patients without a recent history of antibiotic exposure. However, the association was not statistically significant after pooling studies providing adjusted data.
Antibiotic exposure was a significant risk factor in the pediatric inpatient population in our meta-analysis. This finding is consistent with results from the adult population, where antibiotic exposure has been observed to be the most important modifiable risk factor for the development of CDI. ${ }^{9}$ These findings are consistent with the observation that usage of antibiotics can eliminate the natural gut microbiota and establish a favorable environment for C. difficile. ${ }^{21}$ Multiple classes of antibiotics have been independently associated with CDI in the adult population. ${ }^{22}$ In hospitalized pediatric patients, several antibiotic classes were independently associated with CDI. Specifically, carbapenems were identified as a significant risk factor by 2 studies, ${ }^{23,24}$ while aminoglycosides and cephalosporins were identified by only 1 study. ${ }^{24}$ Individual studies included in this systematic review demonstrated significant risks with use of carbapenems, aminoglycosides, and thrd- or fourth-generation cephalosporins (Supplemental Table 2 online). However, none of the individual antibiotic classes were evaluated by at least 3 different studies and were therefore deemed ineligible for the meta-analysis. Although certain antibiotic classes may in fact be independently associated with increased risk for CDI, more studies performed in the pediatric population are needed to further evaluate these associations. Specifically, design of future studies should clarify on the duration of antibiotic or gastric acid suppression treatment and identify specific antibiotic classes used.

Although our findings are consistent with current acceptance of antibiotic exposure as a risk factor for pediatric CDI, the significance of our results should be considered with caution. Due to the limited availability of studies on the risk factors of CDI in pediatric inpatients, our meta-analysis of antibiotic exposure included $<10$ studies for antibiotic exposure. Additionally, our analysis of antibiotic exposure is subject to confounding due to the inclusion of unadjusted studies because few studies provided adjusted data. Furthermore, the 2 studies that provided adjusted data for antibiotic exposure did not demonstrate a significant association. The loss of significance may be attributed to adjustments for age, sex, chemotherapy, and use of PPIs, H2Ras, and steroids.

Previous investigations of gastric acid suppression as a risk factor for pediatric CDI have been conflicting. Biologically, there is strong plausibility for gastric acid suppression as a risk factor for CDI because the loss of acidity may disrupt the normal 
Table 1. Characteristics of Included Studies

\begin{tabular}{|c|c|c|c|c|c|c|c|}
\hline First Author,Year Published & $\begin{array}{l}\text { Study } \\
\text { Location }\end{array}$ & $\begin{array}{l}\text { Study } \\
\text { Period }\end{array}$ & $\begin{array}{l}\text { Study } \\
\text { Design }\end{array}$ & $\begin{array}{l}\text { Sample } \\
\text { Size, No. }\end{array}$ & $\begin{array}{l}\text { Boys, } \\
\text { No. (\%) }\end{array}$ & $\begin{array}{c}\text { Age } \\
\text { Range, y }\end{array}$ & $\begin{array}{l}\text { Diagnostic } \\
\text { Test/Criteria }\end{array}$ \\
\hline Pascarella F, ${ }^{29} 2009$ & Italy & $2005-2007$ & $\mathrm{RCC}$ & 193 & $107(55.4)$ & $1-18$ & Toxin A/B EIA \\
\hline Turco R, ${ }^{12} 2010$ & Italy & 2005-2009 & $\mathrm{RCC}$ & 136 & $86(63.2)$ & $1-18$ & Toxin A/B EIA \\
\hline Nylund CM, ${ }^{3} 2011$ & USA & 1997-2006 & $\mathrm{RC}$ & $10,495,728$ & $4,198,965(40.0)$ & $1-17$ & ICD9 Code (008.45) \\
\hline Guo S, ${ }^{33} 2012$ & China & 2010-2011 & PC & 140 & $98(70.0)$ & $0-18$ & Toxin A/B PCR \\
\hline Hojsak I, ${ }^{24} 2012$ & Croatia & 2006-2011 & $\mathrm{RC}$ & 744 & $379(51.0)$ & $0-18$ & Toxin A/B EIA \\
\hline De Blank P, ${ }^{32} 2013$ & USA & 1999-2011 & $\mathrm{RC}$ & 33,059 & $18,479(55.9)$ & $1-18$ & $\begin{array}{l}\text { ICD9 code }(008.45) \text {, } \\
\text { billing codes }{ }^{a}\end{array}$ \\
\hline Price V, ${ }^{34} 2013$ & Canada & $1995-2004$ & $\mathrm{RC}$ & 341 & $168(49.3)$ & $1-18$ & Culture \\
\hline Brown KE, ${ }^{28} 2015$ & USA & 2008-2012 & $\mathrm{RCC}$ & 458 & $227(49.6)$ & $1-17$ & Toxin B qPCR \\
\hline Santiago $B,{ }^{35} 2015$ & Spain & 2010-2011 & $\mathrm{RC}$ & 250 & $140(56.0)$ & $0-15$ & Culture, Toxin A/B PCR \\
\hline Ciricillo J, 2016 & USA & 2010-2013 & $\mathrm{RC}$ & 55 & $\mathrm{~N} / \mathrm{A}$ & $0-18$ & Toxin A/B EIA, LAMP \\
\hline Finnerty C, ${ }^{37} 2016$ & USA & $1997-2008$ & $\mathrm{RCC}$ & 85 & $57(67.0)$ & $0-18$ & Toxin A/B EIA \\
\hline Karaaslan A, 2016 & Turkey & $2012-2014$ & $\mathrm{RCC}$ & 986 & $552(56.0)$ & $0-18$ & Toxin A/B EIA, CTA \\
\hline Pant C, ${ }^{31} 2016$ & USA & 2012-2012 & $\mathrm{RC}$ & 12,797 & $6,760(52.8)$ & $1-17$ & ICD9 Code (008.45) \\
\hline Daida A, ${ }^{39} 2017$ & Japan & 2003-2012 & $\mathrm{RCC}$ & 108 & $64(59.3)$ & $0-19$ & Toxin A/B EIA \\
\hline
\end{tabular}

Note. N/A, not available; RCC, retrospective case-control; RC, retrospective cohort; NCC, nested case-control; EIA, enzyme immunoassay; DRG, Diagnosis-Related Group; ICD-9, International Classification of Disease, 9th edition; PCR, polymerase chain reaction; CTA, cytotoxicity assay; LAMP, loop-mediated isothermal amplification; qPCR, quantitative polymerase chain reaction. aPresence of billing codes for Toxin A/B EIA and/or PCR, as well as metronidazole (PO or IV) or vancomycin (PO) within the period of 1 day before or 2 days after diagnostic test.

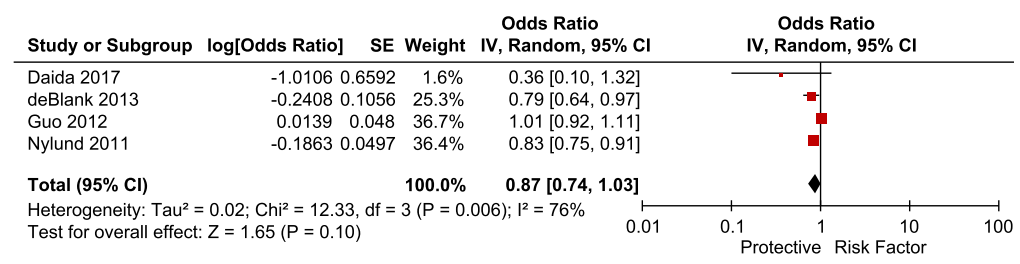

Figure 5. Forest plot of the association between gender and CDI. Vertical line corresponds to no difference point between the 2 groups. Squares correspond to risk ratios. Horizontal lines represent the $95 \%$ confidence intervals. The diamond indicates the pooled relative risk ratios. Note. $\mathrm{df}$, degrees of freedom; $\mathrm{M}-\mathrm{H}$, Mantel-Haenszel.

gastrointestinal microbial diversity ${ }^{25}$ and prolong the survival of spores, ${ }^{26}$ both of which may predispose the host to susceptibility for $C$. difficile. PPI use was significantly associated with development of CDI in the pediatric inpatient population in this metaanalysis, although the effect size was small. Most recently, Oshima et $\mathrm{al}^{27}$ reported a 3 -fold increase in risk of CDI with PPI use in their meta-analysis of pediatric patients. The discrepancies between our results may be explained by differences in our study methodologies. Specifically, Oshima et al included studies that examined community acquired CDI in the outpatient setting and exclusively used raw data from chosen studies that were not adjusted for potential confounding factors. In contrast, we avoided unadjusted data in our analysis of PPI use, and we used only data that had been adjusted by multivariable logistic regression in the original studies. In particular, we obtained unpublished multivariate data for the association of PPI use with CDI from the authors of Brown et $\mathrm{al}^{28}$ to include in this meta-analysis, whereas Oshima et al used unadjusted data from the same study. Nonetheless, the 2 meta-analyses are consistent in demonstrating some degree of association between PPI use and CDI in the pediatric population.

For many of the risk factors assessed by the included studies, we were unable to pool the data due to either an insufficient number of studies or variability in reporting. For example, the presence of IBD as an underlying comorbidity was reported to be a statistically significant risk factor, but was reported by only 2 studies. ${ }^{3,29}$ The association between IBD and CDI might be mediated by the increased use of immunosuppressive agents, antibiotics, and healthcare services, as well as the disruption of the gut mucosal barrier and flora that underlie the pathophysiology of IBD. ${ }^{30}$ Similarly, solid organ transplantation was also reported to be an independent risk factor by 2 separate studies, potentially due to the chronic immunosuppressive therapy in these patients. ${ }^{3,31}$ Malignancies in general as well as specific subtypes of tumors were reported to be independent risk factors in several studies. Again, the association may be mediated by the immunosuppressive and antimicrobial effects of chemotherapy, which was reported to be a statistically significant risk factor itself in 1 study. ${ }^{32}$ In general, additional studies examining these risk factors in the hospitalized pediatric population are needed to validate these findings.

Our meta-analysis has several limitations. First, relatively few studies were identified for our meta-analysis, and they were exclusively observational. To address this limitation, we searched 4 separate databases and included $>2,000$ articles in our original search. Unfortunately, studies of risk factors for CDI in the pediatric population are limited in nature, and they often include community acquired-CDI in combination to hospital acquired-CDI. Due to differences in the acquisition of these 2 conditions, we did not feel that using studies of exclusively outpatient CDI infections would 
be representative of the risk factors for hospitalized patients. As a result, the number of eligible studies was limited in our analysis. Second, contemporary practice guidelines recommend the use of nucleic acid amplification test over the use of other modalities in the diagnosis of CDI. ${ }^{9}$ Due to the paucity of new studies of CDI in pediatric patients, however, most of the studies included in this meta-analysis are older and utilized previously accepted methods such as toxin EIA (Table 1). Third, we evaluated antibiotic exposure as a composite variable without clarifying the specific antibiotics used and duration of treatment because few studies had reported this information. In reality, select antibiotics may have greater effects on CDI compared to others. ${ }^{23}$ Fourth, although our meta-analysis incorporated studies representing $>10$ million patients overall, several studies with large sample size, particularly that of Nylund et $\mathrm{al}^{3}$ did not provide data on important risk factors such as antibiotic exposure and PPI use. A final limitation is the utilization of unadjusted studies that are especially prone to bias and confounding by additional variables. We performed subgroup analyses with only adjusted studies where possible, but we felt the limited number of studies justified the use of unadjusted studies for a preliminary examination of these risk factors. Additional adjusted studies of risk factors for pediatric inpatient CDI would provide the data for a more robust meta-analysis of these risk factors in the future.

In conclusion, we found that antibiotic exposure and PPI use may be risk factors for CDI infection in hospitalized pediatric patients. Clinicians should continue to utilize antibiotics judiciously in hospitalized patients to minimize the risk for CDI, and similar considerations may be beneficial prior to administration of PPIs. Higher-quality adjusted studies of risk factors in the pediatric population with better defined study parameters and definitions for risk factors are needed to validate these results and to further explore other potential risk factors, including the risk associated with specific antibiotic classes.

Supplementary material. To view supplementary material for this article, please visit https://doi.org/10.1017/ice.2019.23

Author ORCIDs. Scott Anjewierden, (D) 0000-0001-5542-1599; Chaitanya Pant, (D) 0000-0003-4599-7644; Abhishek Deshpande, (D) 0000-0001-55222995

\section{Acknowledgments. None.}

Financial support. No financial support was provided relevant to this article.

Conflicts of interest. Abhishek Deshpande has received research support from 3M, Clorox, and STERIS unrelated to this study. All other authors report no conflicts of interest relevant to this article to disclose.

\section{References}

1. Langley JM, LeBlanc JC, Hanakowski M, Goloubeva O. The role of Clostridium difficile and viruses as causes of nosocomial diarrhea in children. Infect Control Hosp Epidemiol 2002;23:660-664.

2. Magill SS, Edwards JR, Bamberg W, et al. Multistate point-prevalence survey of health care-associated infections. N Engl J Med 2014;370:1198-1208.

3. Nylund CM, Goudie A, Garza JM, Fairbrother G, Cohen MB. Clostridium difficile infection in hospitalized children in the United States. Arch Pediatr Adolesc Med 2011;165:451-457.

4. Khanna S, Baddour LM, Huskins WC, et al. The epidemiology of Clostridium difficile infection in children: a population-based study. Clin Infect Dis 2013;56:1401-1406.

5. Sammons JS, Localio R, Xiao R, Coffin SE, Zaoutis T. Clostridium difficile infection is associated with increased risk of death and prolonged hospitalization in children. Clin Infect Dis 2013;57:1-8.
6. Zilberberg MD, Tillotson GS, McDonald LC. Clostridium difficile infections among hospitalized children, United States, 1997-2006. Emerg Infect Dis 2010;16:604-609.

7. Toltzis P, Kim J, Dul M, Zoltanski J, Smathers S, Zaoutis T. Presence of the epidemic North American pulsed-field type 1 Clostridium difficile strain in hospitalized children. J Pediatr 2009;154:607-608.

8. Gupta A, Pardi DS, Baddour LM, Khanna S. Outcomes in children with Clostridium difficile infection: results from a nationwide survey. Gastroenterol Rep 2016;4:293-298.

9. McDonald LC, Gerding DN, Johnson S, et al. Clinical practice guidelines for Clostridium difficile infection in adults and children: 2017 update by the Infectious Diseases Society of America (IDSA) and Society for Healthcare Epidemiology of America (SHEA). Clin Infect Dis 2018;66(7):e1-e48.

10. Sandora TJ, Fung M, Flaherty K, et al. Epidemiology and risk factors for Clostridium difficile infection in children. Pediatr Infect Dis J 2011; 30:580-584.

11. Dulęba K, Pawłowska M, Wietlicka-Piszcz M. Clostridium difficile infection in children hospitalized due to diarrhea. Eur J Clin Microbiol Infect Dis 2014;33:201-209.

12. Turco R, Martinelli M, Miele E, et al. Proton pump inhibitors as a risk factor for paediatric Clostridium difficile infection. Aliment Pharmacol Ther 2010;31:754-759.

13. McFarland LV, Brandmarker SA, Guandalini S. Pediatric Clostridium difficile: a phantom menace or clinical reality? J Pediatr Gastroenterol Nutr 2000;31:220-231.

14. Moher D, Liberati A, Tetzlaff J, Altman DG, PRISMA Group. Preferred reporting items for systematic reviews and meta-analyses: the PRISMA statement. Ann Intern Med 2009;151:264-269.

15. Wells G, Shea B, O'Connell D, et al. The Newcastle-Ottawa Scale (NOS) for assessing the quality of nonrandomised studies in meta-analyses. Ottawa Health Research website. http://www.ohri.ca/programs/clinical_epidemiology/ oxford.asp. Published 2000. Accessed January 23, 2019.

16. DerSimonian R, Laird N. Meta-analysis in clinical trials. Control Clin Trial 1986;7:177-188.

17. Deeks JJ, Higgins JP, Altman DG. Analysing data and undertaking metaanalyses. In: Higgins JPT and Green S (editors). Cochrane Handbook for Systematic Reviews of Interventions. New York: John Wiley \& Sons; 2008. Pp. 243-296.

18. Duval S, Tweedie R. Trim and fill: a simple funnel-plot-based method of testing and adjusting for publication bias in meta-analysis. Biometrics 2000;56:455-463.

19. Kim J, Smathers SA, Prasad P, Leckerman KH, Coffin S, Zaoutis T. Epidemiological features of Clostridium difficile-associated disease among inpatients at children's hospitals in the United States, 2001-2006. Pediatrics 2008;122:1266-1270.

20. Pant C, Anderson MP, Deshpande A, et al. Health care burden of Clostridium difficile infection in hospitalized children with inflammatory bowel disease. Inflamm Bowel Dis 2013;19:1080-1085.

21. Dethlefsen L, Huse S, Sogin ML, Relman DA. The pervasive effects of an antibiotic on the human gut microbiota, as revealed by deep 16S rRNA sequencing. PLOS Biol 2008;6(11):e280.

22. Slimings C, Riley TV. Antibiotics and hospital-acquired Clostridium difficile infection: update of systematic review and meta-analysis. J Antimicrob Chemother 2014;69:881-891.

23. Adams DJ, Eberly MD, Rajnik M, Nylund CM. Risk factors for communityassociated Clostridium difficile infection in children. J Pediatr 2017;186: 105-109.

24. Hojsak I, Ferenc T, Bojanić K, et al. Incidence of Clostridium difficile infection in children with inflammatory bowel disease compared to oncology and immunocompetent patients. Digestion 2012;86:6-11.

25. Seto CT, Jeraldo P, Orenstein R, Chia N, DiBaise JK. Prolonged use of a proton pump inhibitor reduces microbial diversity: implications for Clostridium difficile susceptibility. Microbiome 2014;2:42.

26. Jump RLP, Pultz MJ, Donskey CJ. Vegetative Clostridium difficile survives in room air on moist surfaces and in gastric contents with reduced acidity: a potential mechanism to explain the association between proton pump inhibitors and C. difficile-associated diarrhea? Antimicrob Agents Chemother 2007;51:2883-2887. 
27. Oshima T, Wu L, Li M, Fukui H, Watari J, Miwa H. Magnitude and direction of the association between Clostridium difficile infection and proton pump inhibitors in adults and pediatric patients: a systematic review and meta-analysis. J Gastroenterol 2018;53:84-94.

28. Brown KE, Knoderer CA, Nichols KR, Crumby AS. Acid-suppressing agents and risk for Clostridium difficile infection in pediatric patients. Clin Pediatr (Phila) 2015;54:1102-1106.

29. Pascarella F, Martinelli M, Miele E, Del Pezzo M, Roscetto E, Staiano A. Impact of Clostridium difficile infection on pediatric inflammatory bowel disease. J Pediatr 2009;154:854-858.

30. Sinh P, Barrett TA, Yun L. Clostridium difficile infection and inflammatory bowel disease: a review. Gastroenterol Res Pract 2011;2011:136064.

31. Pant C, Deshpande A, Desai M, et al. Outcomes of Clostridium difficile infection in pediatric solid organ transplant recipients. Transpl Infect Dis 2016;18:31-36.

32. de Blank P, Zaoutis T, Fisher B, Troxel A, Kim J, Aplenc R. Trends in Clostridium difficile infection and risk factors for hospital acquisition of Clostridium difficile among children with cancer. J Pediatr 2013;163: 699-705.e1.
33. Guo S, Xu X, Dong F. Risk factors of Clostridium difficile-associated diarrhea in children. Zhonghua Yi Xue Za Zhi 2012;92:1836-1840.

34. Price V, Portwine C, Zelcer S, et al. Clostridium difficile infection in pediatric acute myeloid leukemia: from the Canadian Infections in Acute Myeloid Leukemia Research Group. Pediatr Infect Dis J 2013;32:610-613.

35. Santiago B, Guerra L, García-Morín M, et al. Clostridium difficile isolation in children hospitalized with diarrhea. An Pediatr 2015;82:417-425.

36. Ciricillo J, Haslam D, Blum S, et al. Frequency and risks associated with Clostridium difficile-associated diarrhea after pediatric solid organ transplantation: a single-center retrospective review. Transpl Infect Dis 2016;18:706-713.

37. Finnerty CC, Herndon DN, Lee JO, et al. Morbidity and mortality in severely burned children with Clostridium difficile-associated diarrhea. Surgery 2016;159:1631-1637.

38. Karaaslan A, Soysal A, Yakut N, et al. Hospital-acquired Clostridium difficile infection in pediatric wards: a retrospective case-control study. SpringerPlus 2016;5:1329.

39. Daida A, Yoshihara H, Inai I, et al. Risk factors for hospital-acquired Clostridium difficile infection among pediatric patients with cancer. J Pediatr Hematol Oncol 2017;39:e167-e172. 